\title{
THE INVARIANCE OF MOLECULAR TOPOLOGY IN CHEMTCAL REACTIONS
}

\author{
EUGENY V. BABAEV \\ Moscow State University \\ Department of Organic Chemistry \\ Moscow 119899 Russia
}

\section{Introduction}

There are two different pictures of molecular structure: the classical and the quantummechanical. The classical picture is naive-empirical and is the chemical one; it is connected with classical structural formulae, ball-and-stick models, the phenomenologica! Lewis concept and the Gillespy rules for prediction of molecular geometry. This picture now endures as the heuristic instrument for the planning of chemical synthesis, for communication between experimental chemists, and for chemical education. The quantum-mechanical picture is the physical one; it is based on the application of quantum mechanical ideas to molecular structures and on quantum-chemical calculations of different degrees of sophistication. Many attempts have been made in theoretical chemistry to find some symbiosis between these two different levels of description of molecular structure; only in recent years the desired compromise seems to have been found in the topological nature of both the quantum-mechanical and classical models of the molecular structure.

Topology is not just graph theory, and similarly chemical topology is not just the use of a graph as an image of a molecular structure or chemical reaction ${ }^{1.2}$ as it is usually considered. ${ }^{3}$ One of the main ideas in classical topology $y^{46}$ is to study spaces which can be continuously deformed into one another, and to find the invariants of such spaces. Some known chemical applications of these ideas (e.g. the topological invariants of surfaces and their critical points) are used to describe electron density maps ${ }^{10}$ or potential energy surfaces ${ }^{\text {s; }}$; some topological invariants of the polyhedrons are also used to understand the electron-counting rules in the chemistry of clusters. ${ }^{7}$ In the cited approaches, the ideas of topology are applied to the quantum-chemical picture of molecular structure. It seems that there is only one work ${ }^{\text {ld }}$ devoted to the topological description of classical structures and the electron-counting rules for usual molecules with localized bonds.

It is the aim of this paper to introduce special spaces, two-dimensional manifolds or surfaces, as new images of molecules with localized bonds, starting only from the classical picture of the molecular structure. One can easily get these surfaces from graphs corresponding to the usual Lewis diagrams of molecules. Some qualitative chemical concepts, which are rather poorly formalized in the language of graph theory, seem to be more clear from the point of view of surface topology. Moreover, because the topological 
invariants of the surfaces are based on the usual chemical electron-counting rules, it seems that the general classical pictures of molecular structures and reactions is closer to manifold topology than to the graph-theoretical description. The suggested approach and its further development seems to be a new branch of interaction between topology and chemistry.

\section{From a Lewis Diagram to the Pseudo-graph and Graphoid}

Consider a Lewis diagram $L(M)=\left(Z, N,\left\{q_{j}\right\}\right)$ of a molecule $M$ with localized bonds ${ }^{k_{s}}$ and with $Z$ valence electrons and $N$ atoms, where the $i$-th atom contains $q_{i}$ valence electrons (for the non-transition elements $q_{i}$ coincides with their group number in the Periodic System). For the given Lewis diagram the unique molecular pseudo-graph (a multi-graph with loops $\left.{ }^{9}\right) G(M)=\left(V, R,\left\{\right.\right.$ deg $\left.\left.v_{\mathrm{b}}\right\}\right)$ can be found, where the number of vertices $V$ is the same as $N$, the number of the edges $R$ is equal to $Z / 2$, the degree of any $i$-th vertex deg $y_{\mathrm{j}}$ is equal to $q_{\mathrm{j}}$, and any loop of the graph $G(M)$ corresponds to a lone pair in the starting diagram $L(M)$ (Chart la). This definition (the importance of which has been discussed earlier from different points of view ${ }^{\text {s.12 }}$ ) connects the Euler equation for a (pseudo)graph" with the vaience electron count in a molecule:

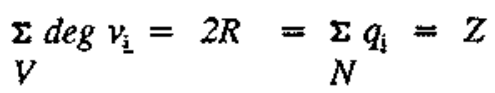

(A somewhat similar definition of a molecular pseudo-graph has been independently used by $\mathrm{Kwasniska}^{\mathrm{t3}}$ in his graph-theoretical approach to organic reactions.)

If the starting molecule contains $Z$ valence electrons and if $L$ of them are unpaired, then the corresponding topological image of a Lewis diagram is no more the pseudo-graph. Let us call a graphoid $G^{\prime}(M)=\left(V, R, L,\left\{\right.\right.$ deg $\left.\left.v_{i}\right\}\right)$ the object which one can get from the (pseudo)graph $G(M)=\left(V+L, R+L,\left\{d e g v_{3}\right\}\right)$ by deleting $L$ free (terminated) vertices but not the edges incident to them. Any graphoid has two sorts of edges, $R$ usual and $L$ hemiedges, as well as two sorts of vertices, $V$ usual and $L$ pricked, i.e., it has as its subset a $(V, R)$-(pseudo)graph ${ }^{14}$. It is obvious that the usual edge of a graph in the topological sense is homeomorphic to the closed interval $[a, b]$, while the hemi-edge (without one vertex or point) in $G^{\prime}(M)$ is homeomorphic to the one-side open interval $[a, b)$. On the Chart $1 \mathrm{~b}$ this type of hemi-edge is shown as the line starting from a vertex to infinity. Because these hemi-edges participate $L$-times in the sum deg $v_{\text {, }}$, the Eq. (1a) for the open-shell molecules and their graphoids should be written as in Eq.(1b):

$$
\underset{V}{\Sigma} \operatorname{deg} v_{\underline{1}}=2 R-L=\sum_{N} q_{1}=Z
$$

We want to mention that in both of the above equations the equality $q_{1}=\operatorname{deg} v_{1}$ for the $i$-th atom is conserved ${ }^{14}$. This means that for any molecule which can be described by more than one Lewis diagram, only one resonance structure (perhaps a non-octet one) should be chosen to construct the pseudo-graph (graphoid) due to this equality. In the case of charged molecules (as well as ylides or betaines) the charges should simply be localized on the appropriate atoms and the necessary number of protons should be added or deleted in these nuclei to get a neutral isoelectronic species with the corresponding 
Chart 1a.

\begin{tabular}{|c|c|c|c|}
\hline Molecule & $\varliminf_{j=0} F_{2}$ & $:_{S F_{4}}$ & $\mathrm{SF}_{6}$ \\
\hline Pseudograph & & & \\
\hline
\end{tabular}

Chart $1 \mathrm{~b}$.

\begin{tabular}{|c|c|c|c|}
\hline Molecule & $\cdot \tilde{N}_{F}$ & : & . \\
\hline Graphoid & & 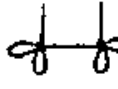 & \& \\
\hline
\end{tabular}

Chart 1c.

\begin{tabular}{|c|c|c|}
\hline $\mathrm{CH}_{3} \ddot{\mathrm{H}}$ & $\mathrm{CH}_{3} \ddot{C H}_{2}$ & ${ }^{+} \mathrm{NH}_{3} \ddot{\mathrm{CH}}$ \\
\hline $\mathrm{CH}_{3} \ddot{O O H}_{2}^{+}$ & & $\mathrm{SiH}_{3} \mathfrak{\mathrm { HH }}_{2}$ \\
\hline $\overrightarrow{\mathrm{BH}_{3}} \ddot{\mathrm{O}}_{2}^{+}$ & ${ }_{\mathrm{PH}_{3}} \ddot{\mathrm{CH}_{2}}$ & $\mathrm{GoH}_{3} \ddot{A} \Leftrightarrow \mathrm{H}_{2}$ \\
\hline
\end{tabular}


change in the $q_{i}$ and $\operatorname{deg} v_{1}$ value." Thus, the isovalent molecules $\mathrm{C}_{2} \mathrm{H}_{3}$ and $\mathrm{CH}_{3} \mathrm{OH}_{3}{ }^{+}$, as well as their isoster ${ }^{-} \mathrm{BH}_{3} \mathrm{OH}_{2}{ }^{+}$and the ylide ${ }^{+} \mathrm{NH}_{3} \mathrm{CH}_{2}{ }^{-}$which are isostructural to the neutral $\mathrm{CH}_{3} \mathrm{NH}_{2}$ after this "charge annihilation," have the isomorphic unlabeled pseudographs (Chart lc).

It is easy to get cyclomatic number $C$ for any connected pseudo-graph $G(M)$ (see the lefthand equality of Eq.(2)). All the loops and the independent cycles between the multiple edges are also included in the cyclomatic number. ${ }^{9}$ For graphoids $G^{\prime}(M) L$ hemi-edges do not participate in any cycle; that is why one should cut them and calculate the $C$-value by using the same equation for the $(V, R)$-subgraph of the $G^{\prime}(M)$. In general, the cyclomatic number for any Lewis diagram has a simple chemical sense as the surn of the (independent) cycles, multiple bonds, and lone pairs, and is determined only by the balanced equation between the number of valence electrons, atoms, and unpaired electrons (see the right-hand equality of Eq. (2)):

$$
C=R-V+I=1 / 2(Z-L)-N+1
$$

\section{From Graph (Graphoid) to Surface}

Consider any (pseudo)graph or graphoid to be in the real three-dimensional space $\mathbf{R}^{3}$. Let us add to any edge and vertex a very small volume of surrounding space. This operation not only conserves completely unchanged the starting graph(oid) structure, but it also adds a new interesting property to the starting object. Now a no-dimensional boundary exists between the internal and external parts of a graph in $\mathbf{R}^{3}$. Consider our graph to consist of empty rubber tubes (edges) which are also empty in their cross-sections (i.e. in the internal vertices), but they are closed in the places of the usually terminated vertices and open on the ends of the hemi-edges.

It is obvious that the resulting object is the two-dimensional manifold in $\mathbf{R}^{3}$ or the twodimensional surface $S(M)$ corresponding to the starting Lewis diagram $L(M)$. By a simple continuous deformation one can easily get some canonical form of this surface, e.g. a sphere with $C$-handles and $L$-holes or $S(C, L)$, (see Chart $2 \mathrm{a}, \mathrm{b}$ ). This surface is orientable; it is closed if $L=0$ and open if $L$ differs from zero. It can be found elsewhere that the pair $(C, L)$ is quite enough to classify ail non-homeomorphic orientable and connected $\mathbf{R}^{2}$ surfaces. ${ }^{46}$

The connected $\mathbf{R}^{2}$-surfaces $S(C, L)$ can be described by their Euler characteristic $\chi$ which is one of the topological invariants, i.e. it is unchanged on topological deformations. ${ }^{46}$ It is not necessary to make a triangulation of the surface to get the $\chi$ value: it depends only on the number of holes $L$ and handles $C$ (see the left-hand equality of Eq.(3)). The use of Eq. (2) shows that for the starting Lewis diagram $L(M)$ its Euler characteristic $\chi$ depends simply on the balance between $\mathrm{N}$ and $\mathrm{Z}$, (see the right-hand equality of Eq. (3)):

$$
x=2-2 C \cdot L=2 N \cdot Z
$$

\section{What is the Topological Homeomorphism from the Chemical Point of View?}

The resulting map $L\left\{Z, N, L,\left\{q_{y}\right\}\right)=>G^{\prime}\left(V, R, L,\left\{\right.\right.$ deg $\left.\left.v_{i}\right\}\right) \Rightarrow S(C, L)$ distributes all the Lewis diagrams on the homeomorphism of their surfaces $S(M)$ on equivalence classes. 
Chart 2a.

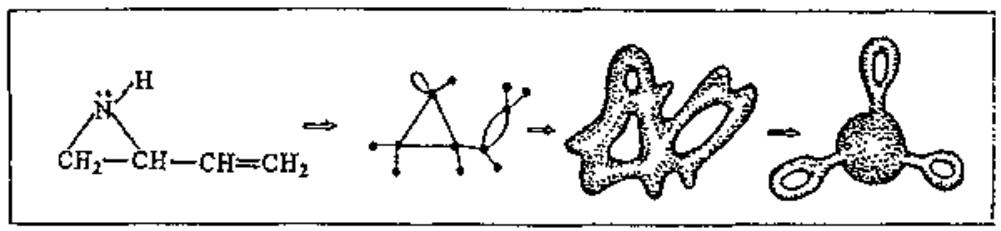

Chart $2 \mathrm{~b}$.

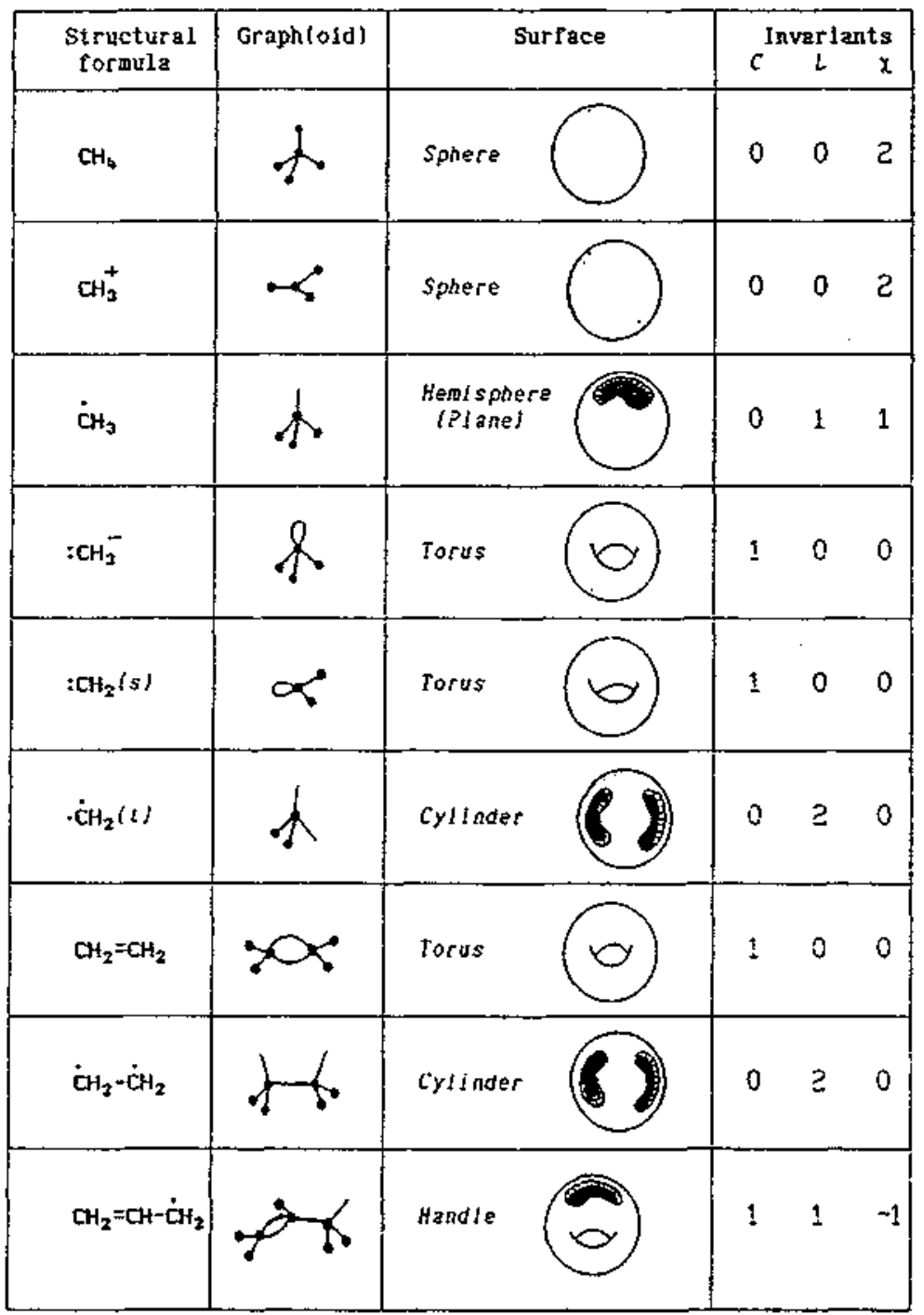


Topological homeomorphism in mathematics is a very crude property showing a similarity of surfaces; nevertheless, this type of topological identity seems to be the important one as a first step in understanding the similarity in a geometrical sense. Following this analogy, it is interesting to compare the homeomorphism of Lewis diagrams as a crude test of the similarity of the structures and chemical behaviors of the corresponding molecules.

There are some general empirical types of chemical similarity both of organic and inorganic molecules (see references 11 and 12) which are based on the usual isoelectronic or $\pi$-isoelectronic analogies, isostructural and homological series, etc., for example:

a) isovalent molecules differing only by the number of the period of any atom in the molecule (e.g. $\mathrm{CH}_{3} \mathrm{NH}_{2}-\mathrm{SiH}_{3} \mathrm{NH}_{3}-\mathrm{CH}_{3} \mathrm{PH}_{2}-\mathrm{SiH}_{3} \mathrm{PH}_{2}-\mathrm{GeH}_{3} \mathrm{AsH} \mathrm{H}_{2}$ etc.);

b) isovalent molecules differing in charge (e.g. $\mathrm{H}_{3} \mathrm{O}^{+}-\mathrm{NH}_{3}-\mathrm{CH}_{3}$ );

c) isosters (alkanes-borazines; $\mathrm{CO}-\mathrm{N}_{2} ; \mathrm{CO}_{2}-\mathrm{N}_{2} \mathrm{O}$ etc.);

d) any number of the resonance structures;

e) all types of tautomers and isomers;

f) classical homologs, differing by one or more $\mathrm{CH}_{2}$-group;

g) $\pi$-isoelectronic molecules with the same number of $\pi$-electrons (e.g. pyrol benzene - borepine, or "pseudoazulenes": azulene - indolizine - pyrrolo, ${ }^{1,2}$ aimidazole);

h) $\pi$-isoelectronic molecules with the same number of $\pi$-electrons differing in the charge (cyclopentadienyl-anion-benzene-tropilium-cation);

i) members of isostructural series of boron hydrides differing in the BH-fragment (isostructural closo-, nido- or arachno- series, see reference 8b).

All the members of each of these series a) - i) have topologically identical (homeomorphic) Lewis diagrams.

It should be mentioned that the homeomorphism in the series a) - e) simply follows our definition of $L(M), G^{\prime}(M)$, and $S(M)$ (Chart 1c), while the topological identity of the molecules in the series f) to i) (differing by the well-known homological fragments $-\mathrm{CH}_{2^{-}}$, $-\mathrm{BH}-$, and $-\mathrm{CH}^{+}$) proves that the concept of the homeomorphism is a very natural and reasonable one for further chemical applications.

It is a well-known phenomenon in mathematical chemistry that some properties of molecules are very similar when the topological indices of their molecular graphs (e.g., the Randić, Hosoya, or Wiener number) coincide. ${ }^{14,215}$ Corresponding to this rule, $\chi$ should be considered as some global (in comparison with the other indices) index of the molecular structure. Its degeneration [or the topological identity of $S(M)$ ] corresponds to some global chemical similarity of the molecules. This is the case for the examples of the series a) - i) mentioned above. Another example is the well-known empirical chemical analogy between lone pair, double bond and 3.4 membered cycles'; this fact should correspond to the topological homeomorphism of these structural fragments to a torus.

On the other hand, the difference in the $x$-value [or in the genus of the closed surfaces $S(M)$, i.e., the number of handles $C$ for the non-radicals] permits us to classify the nonhomeomorphic types of molecules in a linear order as is usual for orientable surfaces in topology. ${ }^{4 \cdot 6}$ The simple chemical sense of the $C$-number is clear: it is a generalization 
to any inorganic compound of the common organic-chemistry idea of the degree of saturation. For any homological $\mathrm{C}_{\mathrm{n}} \mathrm{H}_{2 \mathrm{n}+\mathrm{x}}$ series $\chi$ it is simply its Euler characteristic; any molecule could be more saturated, not only by hydrogenation, but also by coordination with an electrophile.

\section{The Invariance of the Euler Characteristic in Chemical Reactions.}

In the classical Lewis concept of the two-electron and two-centered bond there are only two possibilities to form or to break the bond: The homolytic and the heterolytic. In the simplest case the hydrogen molecule [for which $S(M)$ is a sphere, $\chi=2$ ] could be formed from two atoms (each of which is homeomorphic to a sphere without a point or to a hemisphere, $x=1$ ) or from a proton and a hydride ion (a sphere, $x=2$, plus a torus, $\chi=0$ ). From the surface topology point of view it means gluing the surfaces to a sphere in this manner: to glue the 1-dimensional cycles of the hemispheres in the first case, or to glue the sphere into the hole of the handle in the second case. It is important that in the both operations the Euler characteristic $x$ is the additive value. Other examples also prove this consideration (Chart 3). This principle can be generalized to be the Main Theorem.

\section{The Main Theorem}

The total Euler characteristic of the Lewis diagrams with localized bonds stays unchanged in chemical reactions.

\subsection{Proof}

Consider an ensemble of $K_{s}$ molecules $\left(N_{4}, A_{4}\right.$, and $L_{4}$ are the general number of atoms, and valence and unpaired electrons, in the ensemble) which transforms during the chemical reaction to a new ensemble of the $K_{\mathrm{r}}$ molecules (where $s$ and $f$ indicate starting and final) with corresponding values $N_{f}, A_{t}$ and $L_{p}$. The non-connected graphs (graphoids) with $K_{2}\left(K_{t}\right)$ components and corresponding $V_{2}$ and $R_{1}\left(V_{f}\right.$ and $R_{f}$ ) are determined as mentioned above for the Lewis diagrams of the starting and final ensembles corresponding to Eqs. (1a) and (1b).

For any non-connected graph with $K$ components, Eq. (2) should be changed to Eq. (4) [see the left-hand equality of Eq. (4)],' and after mapping from the graph to the surface with $\mathrm{K}$ components $\mathrm{s}^{\text {s- }}$ the left-hand equality of Eq. (3) should be changed to Eq. (5):

$$
\begin{aligned}
& C=R-V+K=1 / 2(Z-L)-N+K \\
& x=2 K-2 C-L
\end{aligned}
$$

The resulting Euler characteristic $\chi$ for the ensemble of the molecules after the combination of the Eq. (5) with Eq. (4) is equivalent to the right-hand equality of Eq. (3):

$$
\begin{aligned}
x & =2 K-2 C-L=2 K-2[1 / 2[Z-L)-N+K]-L= \\
& =2 K+Z+L+2 N-2 K-L=2 N-Z
\end{aligned}
$$


Chart 3.

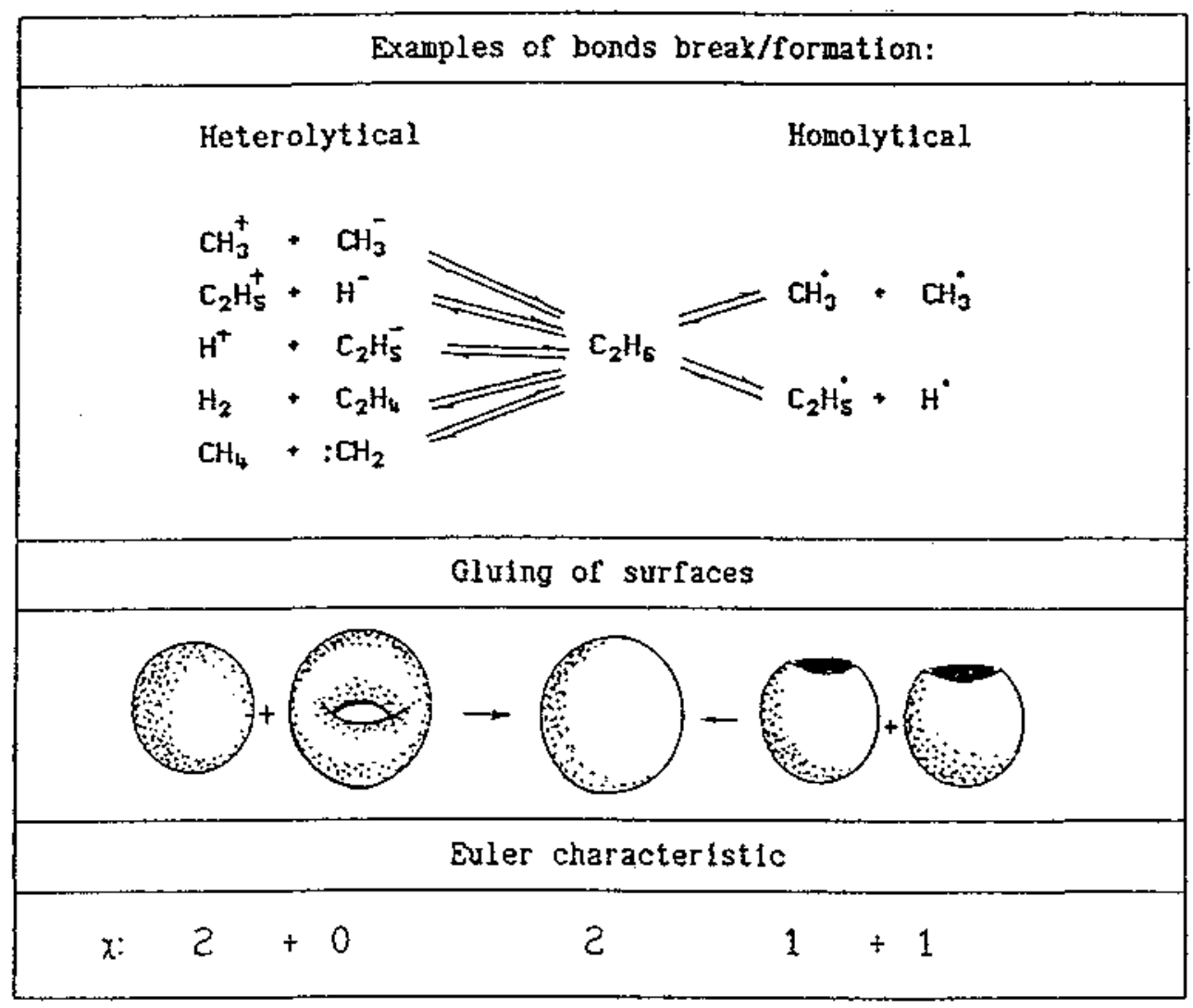


Comparing the values $\chi_{i}$ and $\chi_{t}$ for the starting and final ensembles of the molecules one can easily get Eq. (6):

$$
\Delta \chi=\chi_{\underline{t}}-\chi_{\mathrm{z}}=2\left(N_{\mathrm{f}}-N_{\mathrm{l}}\right)-\left(Z_{\mathrm{f}}-Z_{\mathrm{g}}\right)=0
$$

which is equal to zero due to the conservation of the valence electrons and the atoms in a chemical reactions. Thus, the Main Theorem is proved.

\subsection{Discussion}

The principle corresponding to the Main Theorem we call the conservation of molecular topology in chemical reactions. It is of interest that the conservation of the pure topological property $\chi$ in classical chemistry follows from the conservation of $N$ and $Z$, i.e., from the physical conservation of matter and charge. One can say that an imaginary space with classical chemical structures is mapping to itself during the chemical reactions.

The invariance of $\chi$ is not dependent on the changes of neither the number of molecules $(\Delta K)$, nor on the unpaired electrons $(\Delta L)$, nor the sum of the lone pairs, multiple bonds or cycles (the degree of saturation, $\Delta C$ alone). Because all the members of the triad $(C, L, K)$ are topological invariants in the surface topology, the combination of Eqs. (5) and (6) gives $\mathrm{Eq}$. (7), which is an important chemical consequence:

$$
\Delta x=2 \Delta K-2 \Delta C-\Delta L=0
$$

It follows from Eq. (7) that only five types of interconversions of topological invariants $(K, C, L)$ are permitted in chemical reactions for molecules with localized bonds:

$$
\begin{aligned}
& \Delta L=2 \Delta K \\
& \Delta C=\Delta K \\
& \Delta L=-2 \Delta C \\
& \Delta L=2(\Delta K-\Delta C) \\
& \Delta K=\Delta L=\Delta C
\end{aligned}
$$

(where $\Delta$ corresponds to the difference between the final and starting parameters). All the possible types are symbolically shown on the Chart 4.

All of Eqs. (7a) - (7e) simply follow from the Eq. (7): when one member of the triad $(C, L, K)$ is conserved in a reaction, the two others interconverse according to Eq. (7); the conservation of only the two parameters (i.e. the sudden appearance or disappearance of only one invariant) is impossible in chemistry. For instance, a handle (i.e., lone pair, double bond, or cycle) can appear in a chemical reaction from any of the following:

a) the immediate disappearance of only two holes [see Eq. (7c)]), corresponding in chemistry to intramolecular radical recombination, including triplet-singlet transformations of biradicals, 
Chart 4.

\begin{tabular}{|c|c|c|c|c|}
\hline \multirow{2}{*}{$\begin{array}{l}\text { Interconversed } \\
\text { invariants }\end{array}$} & \multirow{2}{*}{$\begin{array}{l}\text { Conserved } \\
\text { Invar lants }\end{array}$} & \multirow{2}{*}{$\begin{array}{l}\text { Balence } \\
\text { Eqm. }\end{array}$} & \multicolumn{2}{|c|}{ Examples } \\
\hline & & & Chemical equat ion & Gluing of sur races \\
\hline$x, 2$ & $c$ & (72) & $\dot{X}+\dot{Y}=X-Y$ & \\
\hline$x, c$ & $L$ & $\{7 \mathrm{~b}\}$ & $\ddot{X}^{(-)}+Y^{(+)}=X-Y$ & \\
\hline$L, C$ & $x$ & (7e) & $\dot{X} \quad \dot{Y}=X-Y$ & \\
\hline$x, b, c$ & - & (7dd & & \\
\hline - & $x, b, c$ & (7e) & $X_{Y}^{(-)}: Y^{(A)}=X-Y$ & \\
\hline
\end{tabular}


b) the immediate appearance of a new component [see Eq. (7b)], e.g., conversion of alkanes to cycloalkanes,

c) corresponding changes in the number of holes and components [see Eq. (7d)], e.g. , formation of cyclobutane from two triplet ethylenes,

d) the appearance another handle; the appearance of a handle from nothing is forbidden. A good example is the well-known cycle-chain tautomerism: it only seems that a cycle is built from a chain. The cycle which is usually formed, e.g., from an electrophile-nucleophile interaction, has already existed in the "chain" as a lone pair (i.e., hidden cycle) on the nucleophilic center.

The suggested five types of the conservation and interconversion of the topological invariants are good starting points for the further topological classification of chemical reactions. Each type should be subdivided to the different classes, e.g., on the redistribution of the invariants between different surfaces, following to the size of cycles, etc.

\section{Conclusion}

The discussed novel approach could be considered as the first step in our program of "topologization of chemistry" starting from a classical, and not quantum-mechanical, point of view. This gives possibility for physicists to better understand the logic of classical chemistry; for chemists to prove once more that chemistry is not only a descriptive science, but also an exact science; and for mathematicians to find new fields of application. In our further communications we intend to apply some other ideas of manifold topology (fundamental and homology groups, topological images of bypergraphs, etc.) to other classical concepts of chemistry (localization and delocalization, conjugation and hyperconjugation, $\pi$-rich and $\pi$-deficient molecules).

\section{Acknowledgements}

I thank my colleagues, chemists at the Lomonosov University in Moscow, for the third Lomonosov award, which was awarded for this work. I also thank topologists Professor A. T. Fomenko (Moscow, Russia), Professor H. Zieschang (Bochum, BRD) and physicist Professor R. Hefferlin (Collegedale, TN, USA) for fruitful discussions.

\section{References and Notes}

1. a) R.B. King, ed., Chemical Applications of Topology and Graph Theory; Studies in Physical and Theoretical Chemistry, Vol. 28 (Elsevier, Amsterdam, 1983); references are from the Russian translation (Mir Publ., Moscow., 1987); b) R.F.W. Bader, p. 54 in ref.1a; c) P.Mezey, p. 91 in ref.1a; d) M.J. McGlinchey, Y. Tal, p. 148 in ref. la.

2. A. T. Balaban, ed., Chemical Applications of Graph Theory (Academic Press, London, 1976).

3. Book Review: P. Mezey, J. Comput. Chem., 12(1991)139 about R. E. Merrifield, H. E. Simmons, Topological Methods in Chemistry (Wiley Interscience, New York, 1989).

4. H. Seifert, W. Trelfall, A Textbook of Topology, Pure and Appl. Math. Ser., 1980.

5. P. J. Giblin, Graphs, Surfaces and Homology: An Introduction to Algebraic Topology, 2nd edn., (Chapman and Hall, New York, London, 1981). 
6. A. Mishchenko, A. Fonmenko, A Course of Differential Geometry and Topology (Transl. from Russ., Mir Publ., Moscow, 1988).

7. D. M. P. Mingos, R.L. Johnston, in Structure and Bonding, Vol. 68 (SpringerVerlag, Berlin, Heidelberg, 1987), p. 29. R. B. King, D.H. Rouvray, J. Amer. Chem. Soc. 99(1977)7834; R. B. King, Inorg. Chim. Acta, 116(1986)99; B.K. Teo, Inorg. Chem., 23(1984)1251.

8. a) The discussed models are only molecules with localized bonds. The main ide of the approach is to find topological properties [see for example Eq. (3)], which are independent of the pictorial representation of molecular structure by a graph and which are determined only by the electron count in the molecule, with the aim of further generalization of the approach to delocalized systems.

b) The empirical classification of the family of boron hydrides to closo-, nido-, and arachno- structural types connects with electron-counting rules (see ref.7); it is impossible to construct connected pseudographs of this series and to compare their topology with those for molecules with localized bonds. The use of Eq. (3) opens this possibility; the corresponding $\chi$ values are 2,4 , and 6 .

9. F. Harary, Graph Theory (Addison-Wesley, Reading, MA, 1969).

10. a) E. V. Babaev in Proc. of Conference of young scientists (Moscow Univ., 1986) p. 154 (Russ. Ref. Journal 6B(1987)1107); b) E. V. Babaev in Proceed. of 7th AllUnion Conference: Compufers in Chemical Research (Riga, 1986), p. 210 [Russian Ref. Journal 7A(1987)36].

11. E. V. Babaev in Principles of Symmetry and Systemology in Chemistry, N. F. Stepanov, ed., (Moscow University, 1987) [Chem. Abstr. 109, No. 27902b].

12. E. V. Babaev in History and Methodology of Natural Science: Vol. 35. Philosophical Problems of Chemistry. A. P. Rudenko, ed., (Moscow Univ. Publ., 1988) p. $121-140$.

13. V. Kvasniska, Coll. Czech. Chem. Comm., 48(1983)2097.

14. Values $V, R$ and $\left\{\operatorname{deg} v_{i}\right\}$ are topological invariants of (pseudo)graph and the corresponding values $Z, N$ and $\left\{q_{i}\right\}$ are the chemical invariants. It is impossible, staying only with the concept of classical graphs, to conserve Eq. (1a), which connects these two invariants for the open-shell molecules. In some approaches (e.g. N. S. Zefirov, S. S. Tratch, G. A. Gamiani, Zh. Org. Khim. 22(1986)1341) an unpaired electron is considered as a label (phantom-centre) on the additional terminated vertex, i.e, Eq. (1a) is violated.

15. M.I. Stankevitch, I. V. Stankevitch, N. S. Zefirov, Uspekhi Khimii (Russ.) 57 vol. 3(1977)337.

16. The simplest example of the analogy between lone pairs, double bonds, and small strained cycles is the well-known electrophylic addition reaction, e.g. protonation. We mention that during protonation all the starting structural fragments, homeomorphic to a torus, are transformed to cations, homeomorphic to a sphere. We mention also that this analogy proves itself also for other strained cycles in polycyclic systems, but for larger cycles this analogy has only theoretical interest. 